\title{
On the Performance of Multi-user DS-CDMA Systems over Power-Line Channels
}

\author{
Khaled M. Rabie and Emad Alsusa \\ Microwave and Communication Systems (MACS) Group, \\ School of Electrical and Electronic Engineering, \\ The University of Manchester, United Kingdom, M13 9PL, \\ Emails: \{khaled.rabie@manchester.ac.uk; e.alsusa@manchester.ac.uk\}
}

\begin{abstract}
In this paper we propose to implement directspread code-division multiple access (DS-CDMA) over powerline channels combined with blanking at the receiver to reduce the effect of impulsive noise (IN). Two spreading sequences are investigated in this study, namely, Walsh-Hadamard and polyphase codes. The impact of different loading scenarios, ranging from light- to full-loading, on the probability of blanking error and output signal-to-noise ratio (SNR) performance is examined. In addition, the optimal blanking threshold that maximizes the system performance is considered for various system loadings and under different IN conditions. Results reveal that the proposed technique is able to provide remarkable output SNR improvement relative to the conventional DS-CDMA system. It is also shown that this enhancement becomes more significant when the number of active users is reduced.
\end{abstract}

Keywords-Blanking; impulsive noise; direct-sequence codedivision multiple access (DS-CDMA); Walsh-Hadamard (WH) codes; peak-to-average power ratio (PAPR); poly-phase codes; power-line communications (PLC).

\section{INTRODUCTION}

Unlike frequency-division multiple access (FDMA), where users are multiplexed using orthogonal frequency bands, in CDMA all users can transmit at the same time occupying the entire frequency band. The most popular CDMA approach is the direct-spread CDMA (DS-CDMA) in which the transmitter multiplies each user's data by a distinct code which is also used at the receiver to despread the signal of the associated user. This technology has been mainly implemented in cellular mobile and wireless personal communication systems [1], [2]. Recently, however, DS-CDMA was also proposed for power-line communications (PLC) [3], [4]. For instance, the authors in [4] found that both orthogonal frequency division multiplexing (OFDM) and DS-CDMA are suitable for PLC and that, unlike the former approach, the latter does not need protocols to support multiple access capabilities. To realize DS-CDMA, numerous spreading codes have been introduced in the literature such as pseudonoise (PN), Gold, Kasami, Walsh-Hadamard and poly-phase sequences. In this paper, however, the focus will be on the WH and PP sequences.

Irrespective of the modulation technique implemented, power-line (PL) channels suffer from a number of problems such as the varying impedance of the wiring, cable attenuation, time-varying network topology and noise. Several classifications for noise over PLs have appeared in the literature most of which categorized this noise into background noise (BN) and and impulsive noise (IN) and, generally, agree that IN is the most dominant factor degrading PLC signals [5][7]. IN is widely modeled with the well-know two-mixture Gaussian model, [8], and likewise, it will be adopted in this paper. To reduce the effect of IN in OFDM-based PLC systems, a nonlinear preprocessor is usually applied at the receiver's front-end to null or/and clip the received signal when it exceeds a certain threshold [9]-[11].

Unlike the OFDM-based studies, in this paper, we implement DS-CDMA with blanking at the receiver to combat IN. Two different spreading codes are assessed for various loading scenarios. Therefore, the contribution of this paper is as follows. First, we evaluate the peak-to-average power ratio (PAPR) performance of the DS-CDMA-WH and DS-CDMAPP schemes as well as their impact on the probability of blanking error under various system loadings. In addition, the corresponding output SNR is considered. The final contribution of this paper resides in addressing the blanking threshold optimization problem for the two DS-CDMA schemes. The results reveal that both DS-CDMA-WH and DS-CDMAPP systems exhibit similar performance given same loading features. It is also found that the enhancement offered by the proposed system becomes more significant when the number of active users is reduced.

The rest of the paper is organized as follows. Section II describes the system model. The complementary cumulative distribution function (CCDF) of the PAPR for DS-CDMA with WH and PP codes is investigated in Section III. Section IV studies the impact of these codes on the probability of blanking error for various system loadings. Section V outlines the simulation results of the output SNR as a function of the blanking threshold. Sections VI addresses the blanking threshold optimization problem and evaluates the corresponding maximum achievable output SNR. Finally conclusions are drawn in Section VII.

\section{SySTEM MOdel}

The system model under study is illustrated in Fig. 1. There are $M$ users in the system and the information bits of each user is first mapped into 16QAM symbols $s_{m}$, where $m=[1,2, \ldots, M]$. After that each symbol is spread using the user-specific code $\mathbf{c}_{m}=\left[c_{m}^{(0)}, c_{m}^{(1)}, \ldots, c_{m}^{(K-1)}\right]$, where $K$ denotes the code length. As mentioned in the introduction, two coding sequences are deployed in this paper, namely, Walsh-Hadamard and poly-phase codes, both of which are reviewed briefly below.

\section{- Walsh-Hadamard Sequences (WH)}

In the case of perfect synchronization, any two WH sequences have cross-correlation function of zero. Such a property makes these codes widely used for the down link transmission of CDMA systems. WH sequences are often 


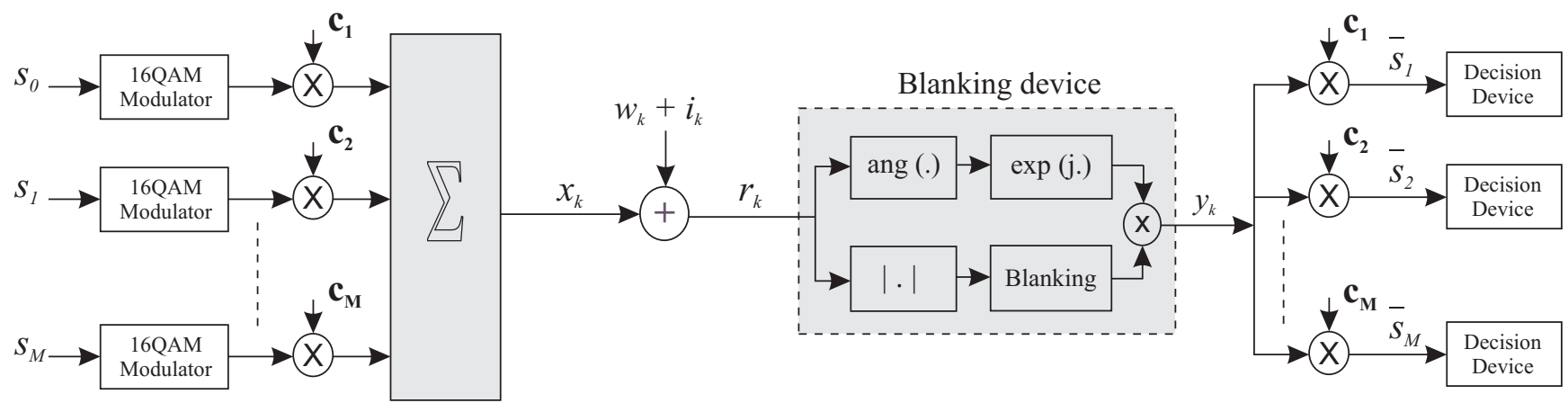

Figure 1: Block diagram of the proposed DS-CDMA system with blanking at the receiver.

defined using the Hadamard matrices $\mathbf{H}_{K}^{w}$ [12] as $\mathbf{H}_{K}^{w}=$ $\frac{1}{\sqrt{2}}\left[\begin{array}{cc}\mathbf{H}_{K}^{w} & \mathbf{H}_{K}^{w} \\ \mathbf{H}_{K}^{w} & -\mathbf{H}_{K}^{w}\end{array}\right]$. The resulting matrix has the property that all the rows are perfectly orthogonal to each other such that

$$
\mathbf{H}_{K} \mathbf{H}_{K}^{T}=K \mathbf{I}_{K}
$$

where $\mathbf{H}_{K}^{T}$ is the transposed Hadamard matrix and $\mathbf{I}_{K}$ is an $K \times K$ unity matrix. The main disadvantage of these sequences is that when the number of users is very small, this yields very large peak power. However, in our investigations we will be using $\mathbf{H}_{64}^{w}$, which is sufficiently large.

- Poly-phase Sequences (PP)

As for PP sequences, they are the non-binary extension of the binary WH sequences. As well as having similar cross-correlation properties as the binary $\mathrm{WH}$, [13], their advantages include robustness against timing misalignment which can dramatically degrade the performance of the binary $\mathrm{WH}$ codes. It is also worth mentioning that, unlike WH sequences, PP codes are not limited by the sequence length and tend to have less PAPR properties as will be shown later. It should be pointed out that, throughout our investigations unless explicitly stated otherwise, we adopt PP codes with four phases $(\phi=4)$.

The spread signals are then multiplexed and transmitted over the PLC channel. This signal is expressed mathematically as

$$
x(t)=\sum_{k=0}^{K-1} \sum_{m=0}^{M-1} s_{m} c_{m}^{(k)}
$$

Using this definition, the PAPR of the transmitted signal is given as

$$
\text { PAPR }=10 \log _{10}\left(\frac{\max |x(t)|^{2}}{\mathbb{E}\left[|x(t)|^{2}\right]}\right)
$$

where $\mathbb{E}[$.$] is the expectation function. As stated earlier, the$ total noise $\left(n_{k}\right)$ over PL channels is composed of $\mathrm{BN}$ and IN

$$
n_{k}=w_{k}+i_{k}, \quad k=0,1,2, \ldots, K-1
$$

where $w_{k}$ is the additive white Gaussian noise (AWGN) and $i_{k}$ is the IN. In this paper we adopt the two-mixture Gaussian model to characterize the IN as [8]

$$
i_{k}=b_{k} g_{k}, \quad k=0,1,2, \ldots, K-1
$$

while $g_{k}$ is complex white Gaussian noise with mean zero and $b_{k}$ is the Bernoulli process with probability $P\left(b_{k}=1\right)=p$; where $p$ denotes the probability occurrence of IN. Therefore, the probability density function (PDF) of the total noise can be written as

$$
\operatorname{Pr}\left(n_{k}\right)=(1-p) \mathcal{G}\left(n_{k}, 0, \sigma_{w}^{2}\right)+p \mathcal{G}\left(n_{k}, 0, \sigma_{w}^{2}+\sigma_{i}^{2}\right)
$$

where $\mathcal{G}($.$) is the Gaussian PDF. \sigma_{w}^{2}$ is the AWGN variance and defines the input SNR as SNR $=10 \log _{10}\left(\frac{\sigma_{x}^{2}}{\sigma_{w}^{2}}\right)$ whereas $\sigma_{i}^{2}$ is the IN variance and defines the signal-to-impulsive noise ratio $(\mathrm{SINR})$ as $\mathrm{SINR}=10 \log _{10}\left(\frac{\sigma_{x}^{2}}{\sigma_{i}^{2}}\right)$. Depending on whether the IN is present or absent, the received signal has one of the following forms

$$
r_{k}=\left\{\begin{array}{ll}
x_{k}+w_{k}, & \mathcal{H}_{0} \\
x_{k}+w_{k}+i_{k}, & \mathcal{H}_{1}
\end{array} \quad k=0,1, \ldots, K-1\right.
$$

The null hypothesis $\left(\mathcal{H}_{0}\right)$ and the alternative hypothesis $\left(\mathcal{H}_{1}\right)$ imply the absence and presence of IN, respectively. At the receiver front-end, blanking is applied as

$$
y_{k}=\left\{\begin{array}{ll}
r_{k}, & \left|r_{k}\right| \leq T \\
0, & \left|r_{k}\right|>T
\end{array} \quad k=0,1, \ldots, K-1\right.
$$

where $T$ is the blanking threshold, $r_{k}$ and $y_{k}$ are the input and output of the blanker, respectively. Determining the optimal blanking threshold (OBT) is the key for achieving best performance. On one hand, using a too low value for $T$ will result in zeroing most of the useful signal and hence degrading performance dramatically. On the other hand, a very large value for $T$ implies that most of the IN energy will be allowed to be part of the detected signal which will also deteriorate performance significantly. Therefore, the blanking threshold must be carefully selected to maximize the performance.

After the noise suppression stage, signal detection takes place as shown in Fig. 1, where the output of the blanker $y_{k}$ is multiplied by the spreading codes $\mathbf{c}_{m}$ to produce estimates for the data symbols of the different users $\bar{s}_{m}$. 


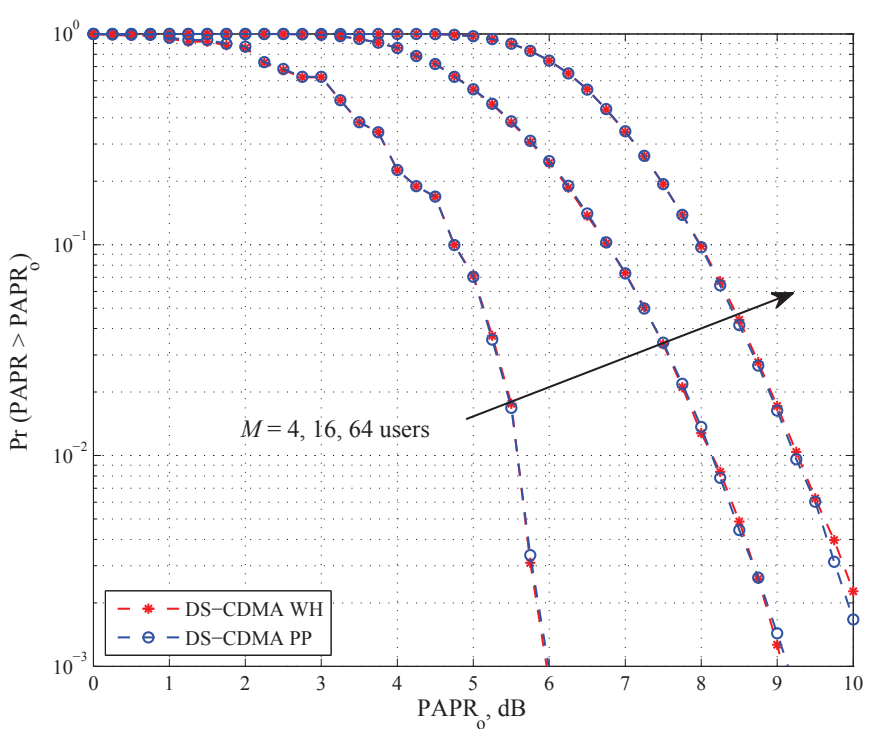

Figure 2: CCDF of the PAPR for the DS-CDMA signals with WH and $\mathrm{PP}$ spreading sequences for various loading scenarios.

\section{Complementary Cumulative Distribution FUNCTION (CCDF)}

The CCDF of the PAPR of a signal defines the probability that the PAPR of a data block exceeds a given threshold $\mathrm{PAPR}_{O}$ and is expressed as

$$
C C D F=\operatorname{Pr}\left(\mathrm{PAPR}>\mathrm{PAPR}_{o}\right)
$$

This parameter is a good measure to predict the blanking efficiency at the receiver as reported in [14] where the authors found that the better the PAPR reduction, the more efficient blanking process is accomplished. Our investigations are based on $\sigma_{x}^{2}=(1 / 2) \mathbb{E}\left[\left|x_{k}\right|^{2}\right]=1, \sigma_{w}^{2}=(1 / 2) \mathbb{E}\left[\left|w_{k}\right|^{2}\right]$ and $\sigma_{i}^{2}=(1 / 2) \mathbb{E}\left[\left|i_{k}\right|^{2}\right]$. Fig. 2 illustrates the CCDF of the DS-CDMA signal with WH and PP sequences for different loading scenarios $(4,16$ and 64 users). It is clearly seen that both spreading codes offer similar CCDF performance. It is also interesting to note that, for both codes, the signal peak is proportional to the number of users. For example, at CCDF of about $10^{-2}$ the PAPR is about $5.5 \mathrm{~dB}$ when the system is lightly-loaded $(M=4)$ whereas for semi-loaded $(M=16)$ and fully-loaded ( $M=64)$ systems, the PAPR is about $8 \mathrm{~dB}$ and $9.25 \mathrm{~dB}$, respectively.

\section{The Probability of Blanking ERror}

In this section we analyze the impact of the number of active users on the probability of blanking error $\left(P_{b}\right) . P_{b}$ is defined as the probability that the amplitude of the received DS-CDMA signal exceeds $T$ when it is not affected by IN and is expressed as

$$
P_{b}=P\left(A_{r}>T \mid \mathcal{H}_{0}\right) P\left(\mathcal{H}_{0}\right)
$$

where $P\left(\mathcal{H}_{0}\right)=(1-p)$. Fig. 3 depicts $P_{b}$ versus $T$ for the DS-CDMA system with WH and PP codes with various loadings $(M=2,4,8$ and 64$)$ when input $\mathrm{SNR}=15 \mathrm{~dB}$. It is apparent from this figure that both spreading codes exhibit similar performances under the same loading condition. As anticipated, it is also seen that the probability worsens, in general, as the number of active users increases due to the high PAPR in such an environment as highlighted previously.

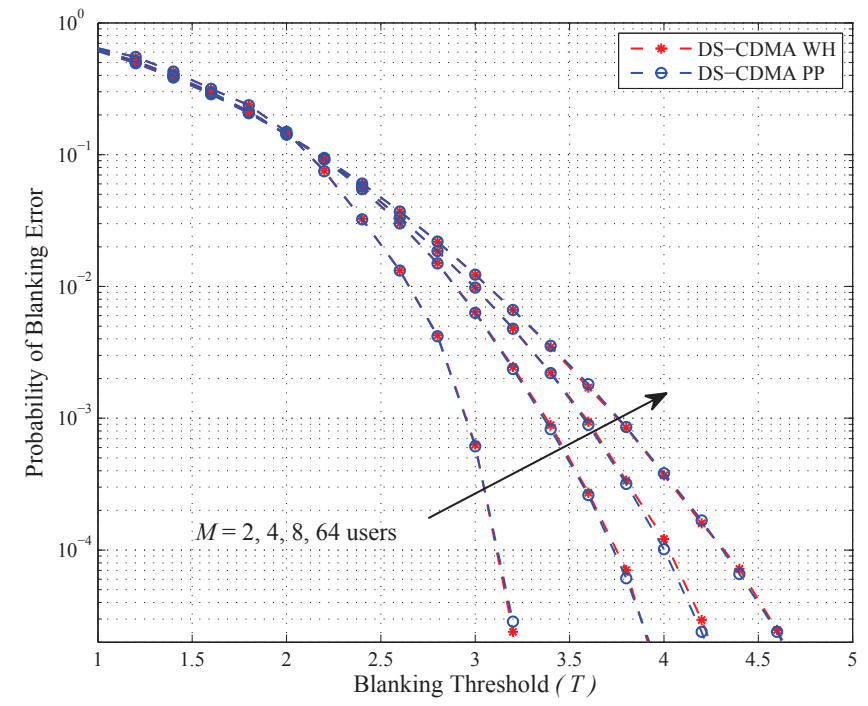

Figure 3: Probability of blanking error versus blanking threshold for the DS-CDMA system with WH and PP spreading codes for various loadings when SNR $=15 \mathrm{~dB}$.

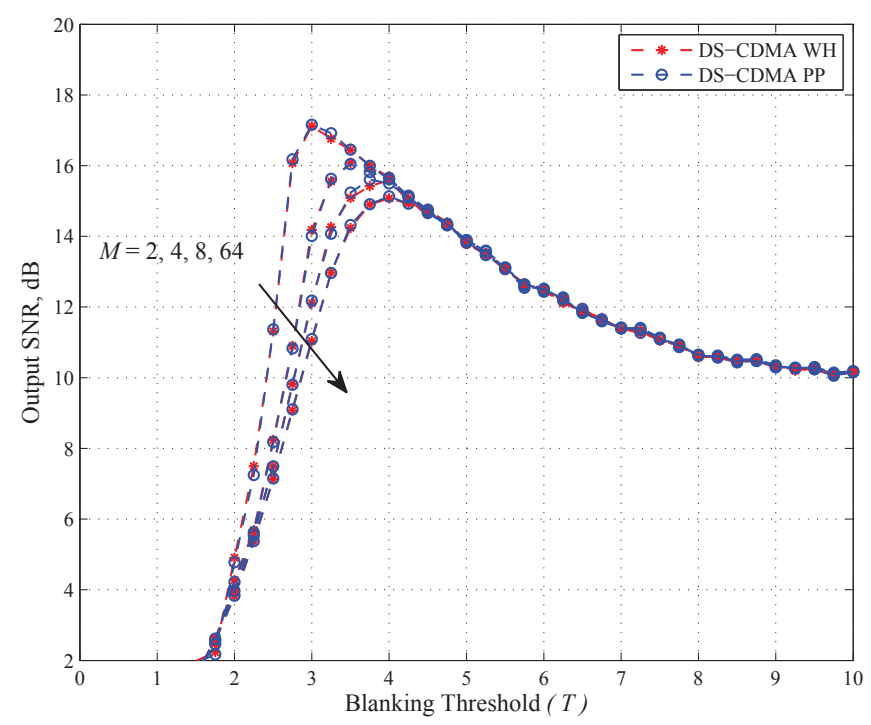

Figure 4: The Output SNR as a function of $T$ for the DS-CDMAWH and DS-CDMA-PP systems with various loading scenarios when SINR $=-10 \mathrm{~dB}, p=0.01$ and $\mathrm{SNR}=25 \mathrm{~dB}$.

\section{The OUtPut SNR VERSUS Blanking THRESHOLD}

This section examines the SNR at the output of the blanking device for the proposed system. It is found as

$$
\mathrm{SNR}_{\text {out }}=\frac{\mathbb{E}\left[\left|x_{k}\right|^{2}\right]}{\mathbb{E}\left[\left|y_{k}-x_{k}\right|^{2}\right]}
$$

Fig. 4 illustrates the output SNR as a function of $T$ for the DS-CDMA-WH and DS-CDMA-PP schemes with various loading conditions when SINR $=-10 \mathrm{~dB}, p=0.01$ and input $\mathrm{SNR}=25 \mathrm{~dB}$. It is noticeable that the application of blanking is always able to improve the system performance regardless of the number of active users if the blanking threshold is chosen carefully. It is also obvious that this improvement becomes less significant as the loading becomes heavier. For example, the SNR gain for a 2-user system can be as high as $7 \mathrm{~dB}$ and about $5 \mathrm{~dB}$ for a fully-loaded system (i.e. 64 active users). Furthermore, it is observed that 


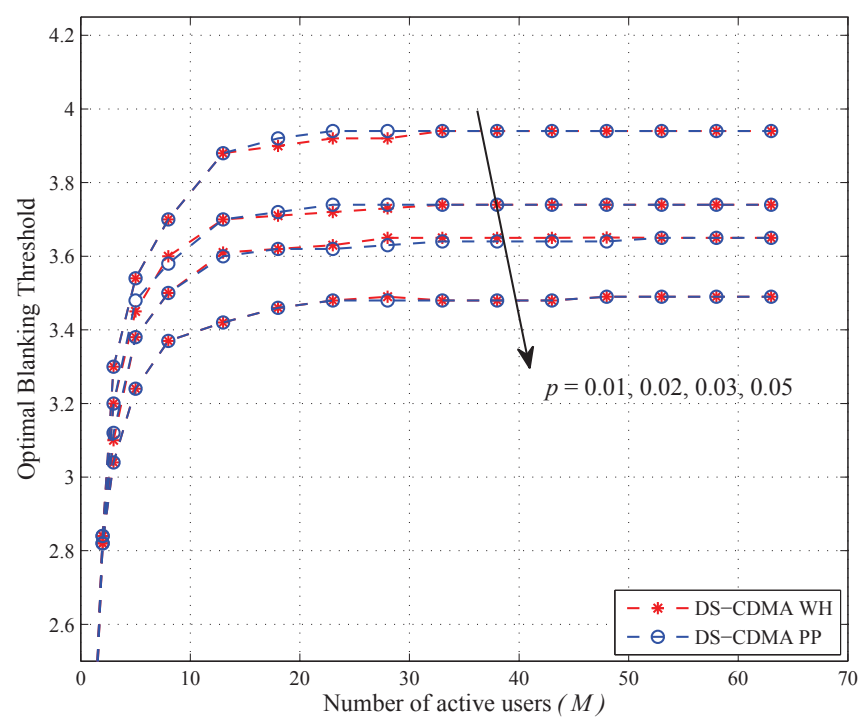

Figure 5: The optimal blanking threshold as a function of $M$ for the DS-CDMA-WH and DS-CDMA-PP systems with different values of $p$ when SINR $=-10 \mathrm{~dB}$ and input $\mathrm{SNR}=25 \mathrm{~dB}$.

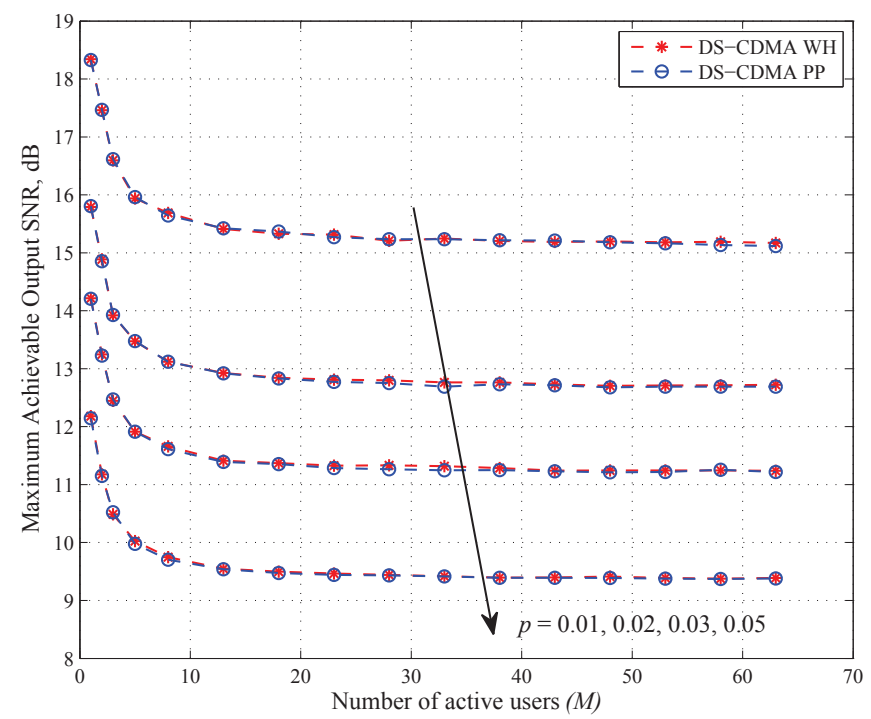

Figure 6: The maximum achievable output SNR as a function of $M$ for the DS-CDMA-WH and DS-CDMA-PP systems with various values of $p$ when SINR $=-10 \mathrm{~dB}$ and input $\mathrm{SNR}=25 \mathrm{~dB}$. when $\{T \rightarrow \infty\}$ (no blanking) the output SNR performance degrades dramatically, approaching $10 \mathrm{~dB}$, due to the that fact that all the IN energy becomes part of the detected signal in which scenario the output SNR can be expressed as $\operatorname{SNR}(T \rightarrow \infty)=10 \log _{10}\left(\frac{\sigma_{x}^{2}}{\sigma_{w}^{2}+p \sigma_{i}^{2}}\right)$. As a final remark on these results, it is clear that for each loading case there exists an OBT that maximizes the output SNR and this will be studied next.

\section{Blanking ThRESHOLD OPTIMIZATION}

Extensive simulations are conducted in this section to find the OBT that maximizes the output SNR for the proposed system. Fig. 5 presents the OBT versus the number of active users for $p=\{0.01,0.02,0.03,0.05\}$ when SINR $=-10$ $\mathrm{dB}$ and input $\mathrm{SNR}=25 \mathrm{~dB}$. In general, it is evident that the OBT is reduced as $p$ becomes higher. For all the IN probabilities, it is shown that in the light-loaded region (i.e. $M \lesssim 20$ ) the OBT is proportional to the number of active users and becomes independent of it when the system is heavily-loaded $(M \gtrsim 20)$.

The maximum achievable output SNR corresponding to the OBT is presented in Fig. 5 versus $M$ for different IN probabilities. The first observation one can see, as expected, is that the performance deteriorates as $p$ becomes higher. In addition, the best performance is obtained when only one user is active and it degrades as the system becomes more loaded. For instance, irrespective of $p$, generally, a SNR gain of up to $3 \mathrm{~dB}$ is achieved for a single-user system relative to that with 10 active users and this enhancement becomes less significant as $M$ approaches 20 users. Furthermore, it is interesting to note that when $(M \gtrsim 20)$ the output SNR tends to level off for all the given IN probabilities.

\section{CONCLUSION}

In this paper we evaluated the performance of DS-CDMA blanking-based systems over PLC channels. Two spreading sequences were exploited, namely, Walsh-Hadamard and poly-phase codes; both of which demonstrated similar performance. The probability of blanking error and the output SNR performances are adopted to characterize the achievable gains of the proposed system. It was found that as the number of active users is reduced the superiority of the proposed system becomes more significant.

\section{REFERENCES}

[1] E. C. Y. Lee, "Overview of cellular CDMA," IEEE Trans. Veh. Technol., vol. 40, no. 2, pp. 291-302, May 1991.

[2] R. L. Pickholtz, B. Milstein, and D. L. Schilling, "Spread spectrum for mobile communications," IEEE Trans. Veh. Technol., vol. 40, no. 2, pp. 291-302, May 1991.

[3] E. Del Re, R. Fantacci, S. Morosi, and R. Seravalle, "Comparison of CDMA and OFDM techniques for downstream power-line communications on low voltage grid," IEEE Trans. Power Del., vol. 18, no. 4, pp. 1104-1109, Oct. 2003.

[4] Y. H. Ma, P. So, and E. Gunawan, "Comparison of CDMA and OFDM systems for broadband power line communications," IEEE Trans. Power Del., vol. 23, no. 4, pp. 1876-1885, Oct. 2008.

[5] D. Middleton, "Non-gaussian noise models in signal processing for telecommunications: new methods an results for class-A and class-B noise models," IEEE Trans. Inform. Theory, vol. 45, no. 4, pp. 1129 -1149 , May 1999.

[6] - "Statistical-physical models of electromagnetic interference," IEEE Trans. Electromagn. Compat., vol. EMC-19, pp. 106-127, Aug. 1977.

[7] — "Canonical and quasi-canonical probability models of class A interference," IEEE Trans. Electromagn. Compat., vol. EMC-25, pp. 76-106, May 1983.

[8] M. Ghosh, "Analysis of the effect of impulse noise on multicarrier and single carrier QAM systems," IEEE Trans. Commun., vol. 44, no. 2, pp. 145-147, Feb. 1996.

[9] O. P. H. et al., "Detection and removal of clipping in multicarrier receivers," Eur. patent appl. EP1043874, Oct. 2000, Bull. 20000/4.

[10] S. V. Zhidkov, "Analysis and comparison of several simple impulsive noise mitigation schemes for OFDM receivers," IEEE Trans. commun., vol. 56, no. 1, pp. 5-9, Jan. 2008.

[11] E. Alsusa and K. Rabie, "Dynamic peak-based threshold estimation method for mitigating impulsive noise in power-line communication systems," IEEE Trans. Power Del., vol. 28, no. 4, pp. 2201-2208, Oct. 2013.

[12] H. F. Harmuth, "Transmission of information by orthogonal functions," Springer-Verlag, Berlin, 1970.

[13] I. Oppenmann, "Orthogonal complex-valued spreading sequences with a wide range of correlation properties," IEEE Trans. Commun., vol. 45, no. 11, pp. 1379-1380, Nov. 1997.

[14] K. Rabie and E. Alsusa, "Preprocessing based impulsive noise reduction for power-line communications," IEEE Trans. Power Del., 2013, Accepted. 